\title{
A study on rate sensitivity of elasto-plastic fracture toughness of TRIP steel evaluated by a small punch test
}

\author{
T. Iwamoto ${ }^{1, a}$, S.-ya Hashimoto ${ }^{2}$, and L. Shi ${ }^{3}$ \\ 1 Faculty of Engineering, Hiroshima University, 1-4-1 Kagamiyama, Higashi-Hiroshima, Hiroshima 739-8527, \\ Japan \\ 2 SUZUKI Motor Corporation, 300 Takatsuka, Minami, Hamamatsu 432-8611, Japan \\ 3 Graduate School of Engineering, Hiroshima University, 1-4-1 Kagamiyama, Higashi-Hiroshima, Hiroshima \\ 739-8527, Japan
}

\begin{abstract}
TRIP steel indicates an excellent characteristic in energy absorption because of its high ductility and strength by strain-induced martensitic transformation (SIMT). Recently, some shock absorption members are being used for automotive industries. For good fuel consumption of the automobile, it would realize the weight reduction without decaying performance if TRIP steel can be applied to those members. It can be considered that the fracture toughness is an important factor to evaluate the performance. To evaluate fracture toughness locally at any point of a product of those members, small punch testing method is quite effective. In the present study, first, an impact small punch testing apparatus is established. In addition, elasto-plastic fracture toughness of TRIP steel under impact loading and its rate sensitivity tested at various deflection rates are challenged to evaluate.
\end{abstract}

\section{Introduction}

Austenitic stainless steel has a metastable austenitic structure and martensitic transformation is occurred from the structure by plastic deformation. This phenomenon is called the strain-induced martensitic transformation (SIMT). A steel with SIMT indicates not only high strength but also high ductility and toughness. This phenomenon which ductility and toughness increase because of SIMT is called transformation-induced plasticity, TRIP. A steel with TRIP is called TRIP steel.

Recently, some shock absorption members are being used for automotive industries. However, a weight of those members increases because a safety standard in world is gradually being strict. Consequently, it is natural that a weight of vehicle should increase because of increasing the strength. Considered recent environmental issues, improving fuel consumption is strongly required for automotive industries. Fuel consumption is deeply related to the weight of vehicles, therefore, the weight reduction is quite essential. If it is possible that TRIP steel is applied to the shock absorption members, it would realize the weight reduction without decaying excellent performance. In order to apply TRIP steel for shock absorption members, a clarification of mechanical and fracture characteristics subjected to impact loading becomes important. Fracture toughness in one of fracture characteristic represents a resistance for crack propagation which involves plastic deformation and causes SIMT in the structure. Thus, it can be thought that evaluating fracture toughness of TRIP steel subjected to impact loading is needed to an application of TRIP steel for such members. In addition, evaluating fracture toughness after manufacturing shock absorption members is necessary to evaluate performance of those members.
In the past, tons of studies about a measurement of fracture toughness under impact loading are conducted. Yokoyama and Kishida [1] evaluated the fracture toughness of aluminum and titanium alloys under impact loading using a split Hopkinson pressure bar (SHPB) method. In the study by Yokoyama and Kishida [1], the newlyproposed 3 point bending impact testing apparatus was established based on the SHPB method. Mutoh et al. [2] evaluated fracture toughness of various steels using the Charpy test method. Shum et al. [3] attempted to evaluate dynamic fracture toughness using compact tension (CT) specimen. However, as these test method requires relatively large spacimen for test preparation, it is difficult to measure the fracture toughness at random point of shock absorption members produced. On the other hand, small punch (SP) test method is developed to evaluate fracture toughness using small specimen for in-service structure of a nuclear power plant. Many researchers attempted to estimate fracture toughness using SP test. For example, Shindo et al. [4] evaluated fracture toughness of stainless steel under cryogenic condition. However, any studies conducting SP test under impact loading cannot be found that any and the test method is not proposed.

In the present study, first, an impact SP testing apparatus is established by combining the SHPB method, which could measure an impact load with a high accuracy and SP test apparatus. Then, a validity of SP testing apparatus is discussed by comparing with the past experimental result. In addition, an elasto-plastic fracture toughness of TRIP steel under impact loading and its rate sensitivity tested at various deflection rates are attempted to evaluate.

\section{Experimental procedure}

\subsection{Specimen}

Specimens used in the present study are aluminum alloy 6061 and AISI304, which is a kind of TRIP steel. The

a e-mail: iwamoto@mec.hiroshima-u.ac.jp This is an Open Access article distributed under the terms of the Creative Commons Attribution License 2.0, which permits unrestricted use, distribution, and reproduction in any medium, provided the original work is properly cited. 
alluminum alloy is used for confirming a validity of SP test apparatus manufactured here. Both specimens are machined to $10 \mathrm{~mm}$ in diameter, $0.5 \mathrm{~mm}$ in thickness with perpendicular to a rolling direction. AISI 304 is subjected to solution heat treatment at $1323 \mathrm{~K}$ for 30 minutes after machining.

\subsection{SP test apparatus and quasi-static SP test}

Figure 1 shows (a) a photograph and (b) a schematic figure of manufactured SP testing apparatus for quasistatic condition. The manufactured apparatus consists of upper and lower dies, and puncher. Specimen is clamped with upper and lower die by screwing four bolts. Puncher is fixed to a frame as shown in this figure to load in the vertical direction as possible. To keep a straightness between centers of the puncher, the specimens and dies, a collar is used as shown figure 1. The experiment is conducted with a conventional material testing machine (Shimazu AG-250kNXplus) at crosshead speeds of 0.2, 2, 20 and $200 \mathrm{~mm} / \mathrm{min}$.

\subsection{Impact SP test based on the drop weight method with the load sensing block}

Figure 2 shows a photograph of the impact test device on the basis of the drop weight method. The specimen is set in the SP test apparatus and the apparatus is placed on the center of the steel plate just above the load sensing block [5]. Then, a weight is dropped with a speed around $5 \mathrm{~m} / \mathrm{s}$ by turning off the electromagnet for a fixation of the weight. Then, the apparatus is loaded impactly by hitting the weight to the top of the apparatus. The load sensing block has two parts. One is a part for the load sensing and the other is a part for the protection of the reflected stress wave into the load sensing part. The load sensing part has a quite small cross-sectional area compared with the other parts because the stress wave reflected at the bottom of the block is hard to propagate back into the load sensing part. To measure the load, two strain gages with a resistance of $350 \Omega$ (Tokyo Sokki Kenkyujo Co., Ltd. UFLA-1-350-11) are glued axisymmetrically at a center of the sensing part. Strain gages are connected to a digital oscilloscope (Yokogawa DL-2700) via a signal conditioner (Kyowa CDV-700A) and the Wheatstone bridge circuit. A gauge line is marked on the top of the puncher and the displacement can be measured by tracking a position of the gauge line using the high speed video camera (Hano Manufacturing Co., Ltd. HNI-1250) with a frame rate of $4000 \mathrm{fps}$.

\subsection{Impact SP test based on the SHPB method}

To conduct an impact SP test acculately, an impact SP test apparatus is established by combining the SHPB method and SP testing apparatus as above-mentioned. Schematic figure of the established apparatus for the impact SP test is shown in figure 3. The established apparatus consists of an air compressor, an air gun, a launcher, a striker bar,

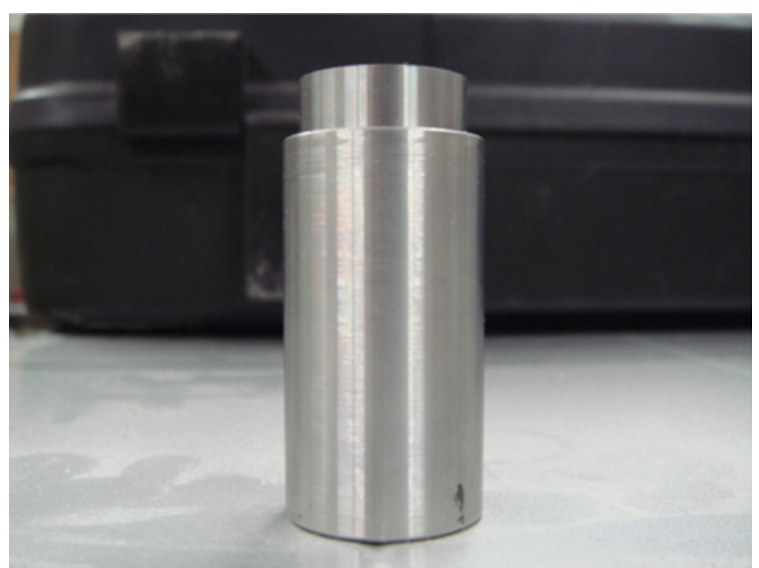

(a)

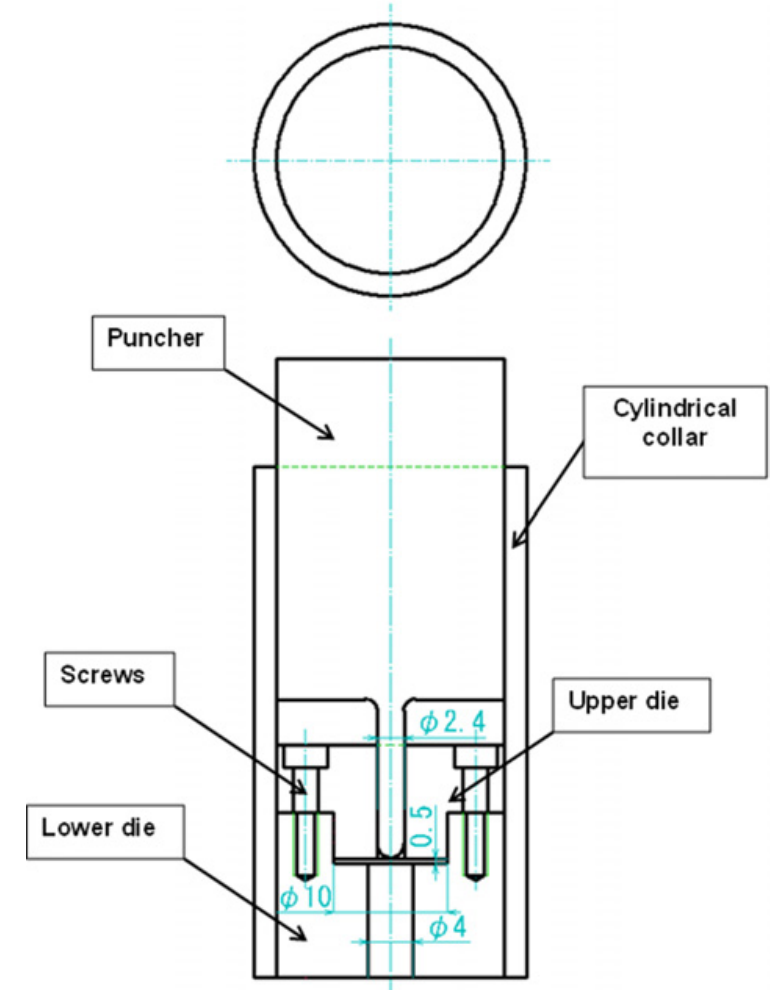

(b)

Fig. 1. (a) Photograph and (b) cross-sectional view of quasi-static SP testing apparatus.

an input bar, an output tube and measurement devices. An internal thread is produced on the edge of the output tube. Another set of the upper die is newly manufactured by providing an external thread in order to connect them to the output tube. The puncher is directly manufactured on one edge of the input bar. To keep straightness between centers of the puncher and the specimen and dies, a collar is used in the same way of quasi-static SP test. The striker bar is launched at a speed of about $10 \mathrm{~m} / \mathrm{s}$ and is hits to the edge of the input bar. To measure the strain pulse, semi-conductor strain gages (Kyowa KSP-2-E4) are glued axisymmetrically at a center of pressure bars. All strain gages are connected to a digital oscilloscope (Yokogawa DL-2700) via signal conditioners (KYOWA CDV-700A) 


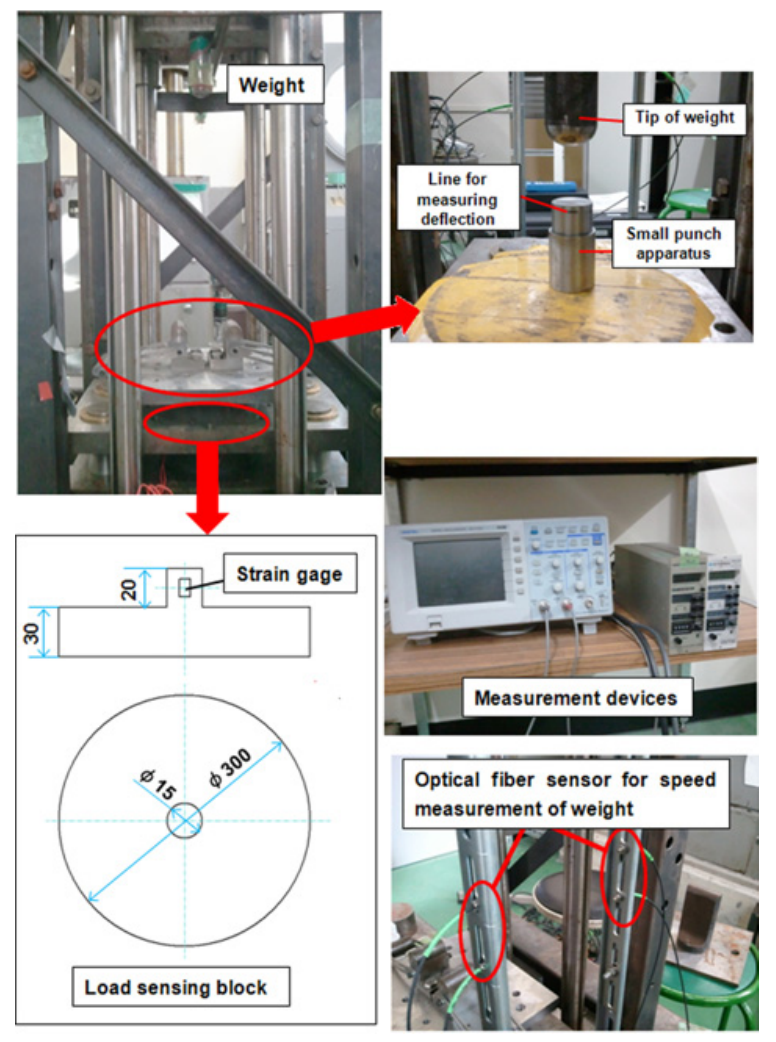

Fig. 2. Weight drop impact testing machine.

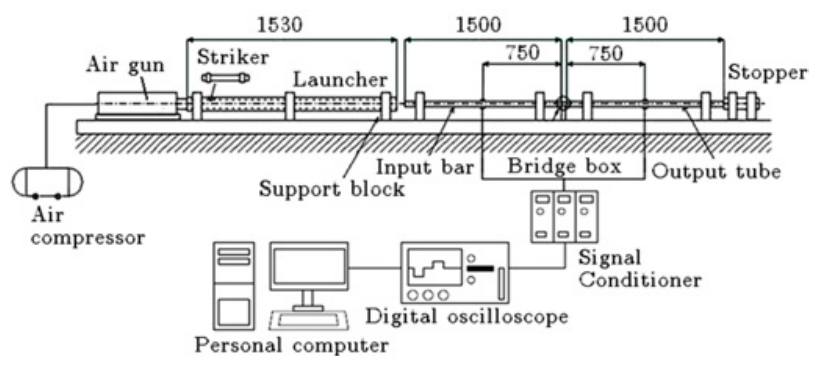

(a)

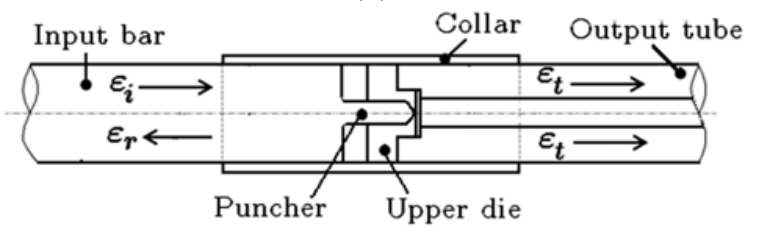

(b)

Fig. 3. Schematic figure of impact SP test apparatus; (a) side view and (b) magnified view near the specimen.

and the Wheatstone bridge circuit. Data are recorded with a sampling rate of $1 \mathrm{MSa} / \mathrm{s}$.

Figure 3(b) shows a magnified figure of figure 3(a) around the specimen. As shown in this figure, when the striker bar hits the input bar, incident strain pulse propagates in the input bar. After that, a part of the incident pulse propagates back into the input bar as reflected strain pulse. Other part of the incident strain pulse propagates into the output bar as transmitted strain pulse. Obeying these strain pulses and one-dimensional wave propagation theory, load

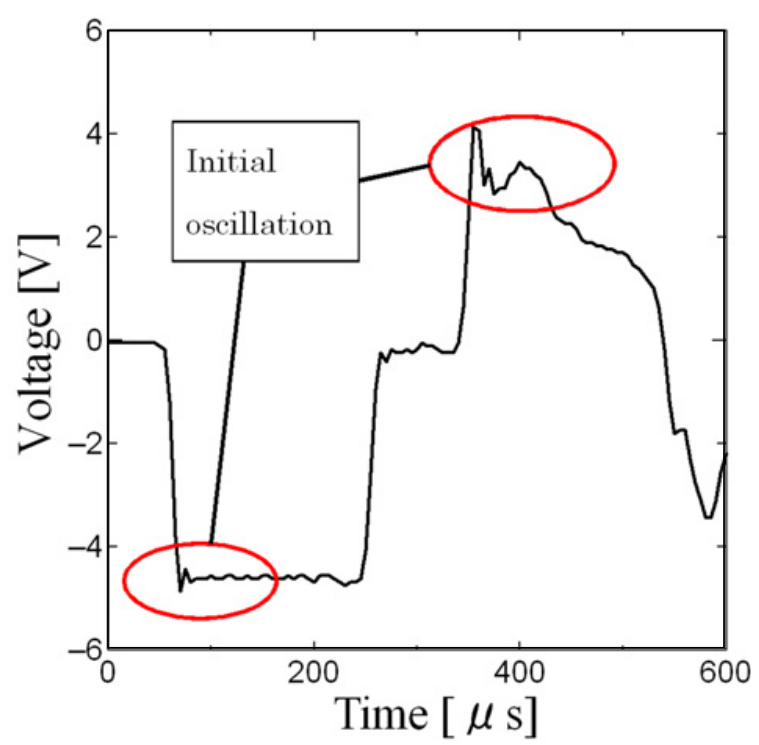

Fig. 4. Initial oscillation on incident and reflected stress waves.

applied to the specimen, particle velocity and deflection can be calculated. The input and output loads $P_{\text {in }}(t)$ and $P_{\text {out }}$ applied to the specimen are calculated as following equation,

$$
P_{\text {in }}(t)=\left\{\sigma_{i}(t)+\sigma_{r}(t)\right\} A_{\text {in }}, P_{\text {out }}(t)=\sigma_{t}(t) A_{\text {out }}
$$

where $A_{\text {in }}$ and $A_{\text {out }}$ are cross-sectional areas of the input bar and the output tubes, $\sigma_{i}, \sigma_{r}$ and $\sigma_{t}$ are the incident, reflected and transmitted stress pulses, respectively. Particle velocities in the input bar and output tube, $v_{\text {inp }}, v_{\text {out }}$ are obtained by the following equation,

$$
v_{\text {inp }}(t)=\frac{\sigma_{i}(t)-\sigma_{r}(t)}{\rho c}, \quad v_{\text {out }}(t)=\frac{\sigma_{t}(t)}{\rho c}
$$

where $\rho$ and $c$ are a mass density and a velocity of longitudinal elastic wave propagation of pressure bars. Using above equation, a maximum deflection at a center of the specimen, $\delta(t)$ is calculated by the following equation,

$$
\delta(t)=\int_{0}^{t}\left\{v_{\text {inp }}\left(t^{\prime}\right)-v_{\text {out }}\left(t^{\prime}\right)\right\} d t^{\prime}
$$

In general, stress wave obtained by SHPB method has initial oscillation as shown in figure 4. To control the oscillation, a pulse shaper is used. The pulse shaper is made of pure copper and the shape is determined as $5 \mathrm{~mm}$ in diameter and $0.3 \mathrm{~mm}$ in thickness. As a result of SP test, load $P$ vs deflection $\delta$ curve can be obtained.

\section{Validity of the established apparatus}

To show validity of the manufactured SP testing apparatus, a quasi-static SP test using aluminum alloy 6061 is conducted at crosshead speed of $0.2 \mathrm{~mm} / \mathrm{min}$ following the previous study [6]. Figure 5 shows a comparison of load - deflection curves obtained in the present study and the previous study [6]. From this figure, both results show fairly good agreement. Thus, it can be said that the manufactured SP testing apparatus for quasi-static test is valid. 


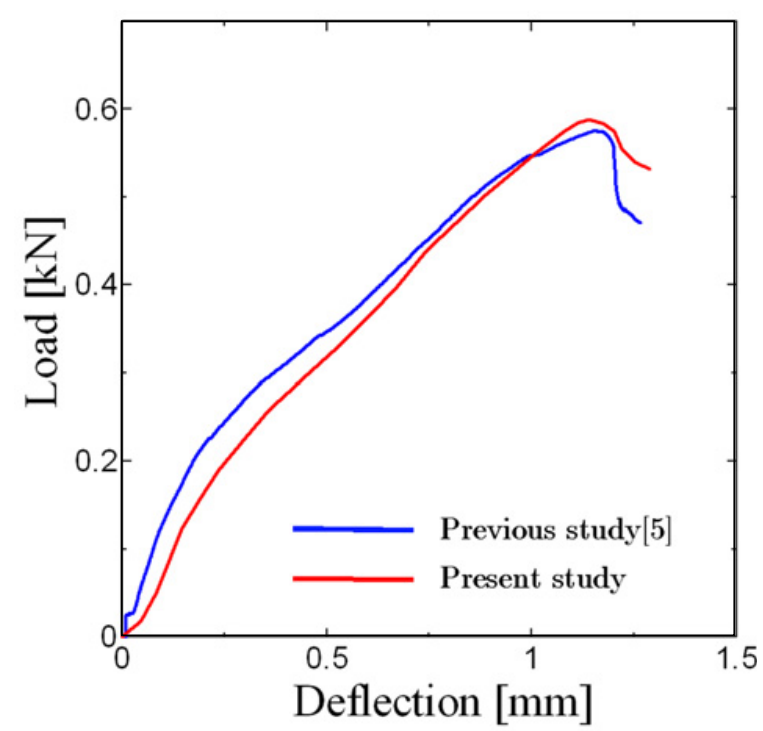

Fig. 5. Comparison of present study with previous study for aluminum alloy 6061 .

\section{Determination of Elasto-plastic fracture toughness by SP test [4]}

Shindo et al. [4] conducted SP test and ASTM E913-81 using CT specimen, and derived an evaluation equation of fracture toughness for SP test. The following shows derivation method of the equation. At first, approximately equivalent fracture strain $\varepsilon_{q f}$ is derived as the following equation.

$$
\varepsilon_{q f}=\ln \left(\frac{t}{t_{0}}\right)
$$

where $t_{0}$ is an initial thickness of specimen and $t$ is a thickness at the fracture. From the correlation between logarithmic equivalent fracture strain and $\ln \left(\delta_{\max } / t_{0}\right), \varepsilon_{q f}$ is determined as following equation.

$$
\varepsilon_{q f}=\alpha\left(\frac{\delta_{\text {max }}}{t_{0}}\right)^{n}
$$

where $\delta_{\max }$ is maximum deflection, and $\alpha$ and $n$ are the material parameters which can be determined by the test condition. From the linear correlation between the above equation and fracture toughness determined by ASTM E813-81, the fracture toughness $J_{I C}$ is determined as the following linear equation.

$$
J_{I C}=A \varepsilon_{q f}+B
$$

where $A$ and $B$ are the parameter which can be identified by the experiments of fracture toughness under different condition. Shindo et al. [4] have already determined the parameters $\alpha, n, A$ and $B$ as shown in Table 1 . However, it is noted that the parameters are determined under a cryogenic condition, which is different from the condition of the present study.

\section{Experimental results and discussion}

Figure 6 shows the stress waves captured from the strain gages during the impact test based on the SHPB method.
Table 1. The parameters determined by Shindo et al. [4] under a cryogenic condition.

\begin{tabular}{llll}
\hline$\alpha$ & $n$ & $A$ & $B$ \\
\hline 0.0756 & 1.83 & 957.7 & 29.5 \\
\hline
\end{tabular}

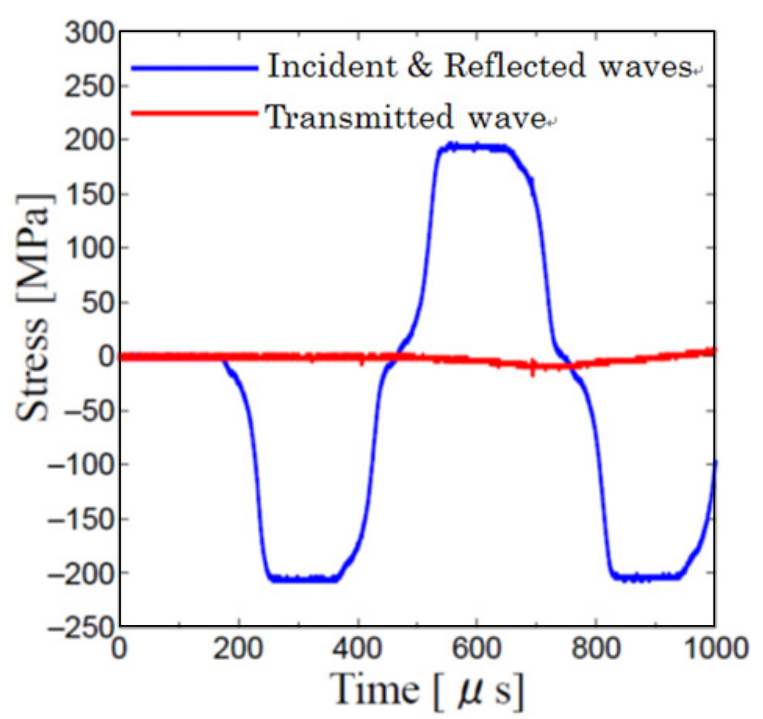

Fig. 6. Stress pulses obtained by the impact SP test based on the SHPB technique.

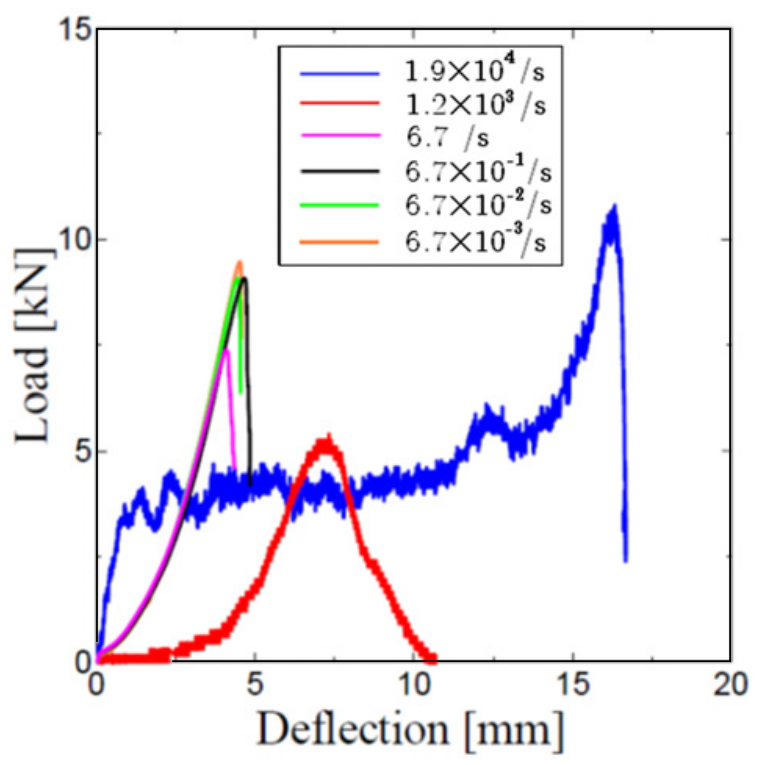

Fig. 7. Load-deflection curves at various deflection rates.

The transmitted wave is quite small compared with the incident and reflected waves since the stress wave becomes quite small at the puncher due to a sudden decrease of the cross-sectional area near the puncher. However, the load and deflection can be calculated by Eq. (1) to (3) because the signal itself can be captured by an appropriate adjustment of the resolution in output voltage.

Figure 7 shows the load-deflection curve at various normalized deflection rates by length of the specimen. As shown in this figure, the deflection at the maximum load increases with a increasing the deflection rate. In the 


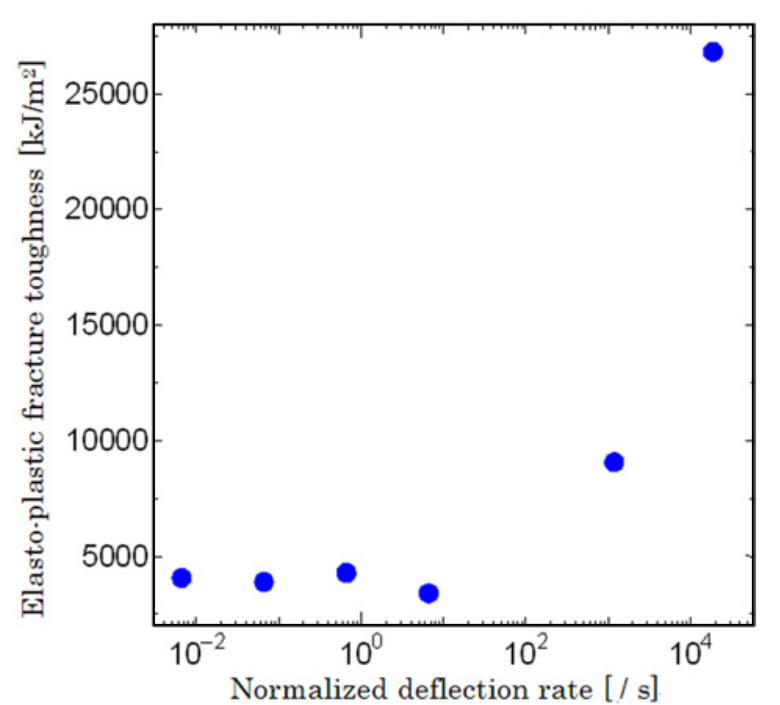

Fig. 8. The semi-logarithmic plot of elasto-plastic fracture toughness-normalized deflection rate.

conventional tensile test for a metallic material, the load increases with an increase of strain rates and maximum strain for failure becomes lower with respect to the strain rate. This implies that a stregth becomes larger, however, a ductlity decreases with respect to the strain rate. The opposite tendency on the rate senstivity of the deflection can be observed here.

Figure 8 shows the semi-logrithmic plot of elastoplastic fracture toughness-normalized deflection rate curve. As shown in this figure, the elastoplastic fracture toughness is increasing exponentially with respect to the deflection rate. At highest deflection rate, the fracture toughness indicates the value over $25000 \mathrm{~kJ} / \mathrm{m}^{2}$ as shown in this figure. It is significantly larger than the values obtained in the previous studies $[2,4]$. The equations expressed in Chapter 4 is defined at the cryogetic temperature, thus, the fracture toughness is overestimated. Therefore, the equations should be re-identified again by experiments at various rate and the rate sentitivity of the fracture toughness should be clarified.

\section{Summary}

Here, first, an impact SP testing apparatus was established by combining the split Hopkinson pressure bar method which could measure impact loading with high accuracy and SP test apparatus. Then, a validity of SP testing apparatus was discussed by comparing with the past experimental result. In addition, an elasto-plastic fracture toughness of TRIP steel under impact loading and its rate sensitivity tested at various deflection rates was attempted to evaluate.

\section{Acknowledgements}

We gratefully acknowledge financial supports from both AMADA foundation and a Grant-in-Aid for Scientific Research from the Ministry of Education, Culture, Sports, Science and Technology, Japan.

\section{References}

1. T. Yokoyama, K. Kishida, Exp. Mech., 29 (1989) 188194.

2. Y. Mutoh, N. Nakaoka, C. Kawamoto, J. Soc. Nav. Arc. Jap., 153 (1983) 329-335.

3. D. Shum, M. N. Bassim, M. R. Bayoumi, Int. J. Fract., 29 (1985) 3-10.

4. Y. Shindo, Y Yamaguchi, K, Horiguchi, Cryogenics, 44 (2004) 789-792.

5. K. Mimura, S. Hirata, Y. Chuman, S. Tanimura, Trans. JSME Ser. A, 62 (1996) 2609-2614. (in Japanese)

6. E. Budzakoska, D. G. Carr, P. A. Stathers, H. Li, R. P. Harrison, A. K. Hellier, W. Y. Yeung, Fatigue Fract. Eng. Mat. Struct., 30 (2007) 796-807. 